\title{
Ambulatory electrocardiographic monitoring in 100 healthy teenage boys
}

\author{
D F DICKINSON, OLIVE SCOTT \\ From the Department of Paediatric Cardiology, Killingbeck Hospital, Leeds
}

SUMMARY Ambulatory monitoring of the electrocardiogram in 100 healthy 14 to 16 year old boys showed heart rates ranging from 45 to 200 beats/minute during the day and from 23 to 95 beats/ minute during sleep. Sinus arrhythmia was present in all cases and was the only variation noted in $17 \%$. Sudden variations in the PP interval occurred in $41 \%$, but a precise diagnosis of the mechanism was usually impossible; $15 \%$ had changes compatible with sinus arrest or temporary complete sinoatrial block, and one boy had a pattern compatible with type II second degree sinoatrial block. Escape rhythms were noted in $26 \%$, first degree atrioventricular block in $12 \%$, and second degree atrioventricular block (Mobitz type I) in 11\%. Mobitz type II second degree atrioventricular block was seen on one occasion in one boy. Ventricular extrasystoles seen in $41 \%$ were of uniform morphology in $75 \%$ and multiform in $25 \%$. Short episodes of ventricular tachycardia were recorded in $3 \%$.

The use of 24 hour recordings of the electrocardiogram is now well established in the investigation of symptomatic individuals suspected of having a cardiac arrhythmia. ${ }^{2}$ In the paediatric age group many patients investigated in this way have no symptoms during the period of recording, but nevertheless a rhythm change is detected. ${ }^{2}$ To assess the importance of these changes a detailed knowledge of the range of rhythm abnormalities found in an unselected asymptomatic population is essential. The findings of studies of 24 hour rhythm patterns in neonates ${ }^{34}$ and young children ${ }^{56}$ have been reported, and differences from the patterns seen in an adult population ${ }^{78}$ have been noted. We report the 24 hour rhythm patterns of a group of healthy teenage boys.

\section{Patients and methods}

Recordings were made for two consecutive periods of 24 hours in 100 boys aged 14 to 16 years from two local schools. Each boy was questioned and examined by one of us (OS). None had a history of syncopal episodes, and no cardiac abnormality was detected in any boy. The technique of recording has been described. 5 The boys carried out their normal

Requests for reprints to Dr D F Dickinson, Killingbeck Hospital, York Road, Leeds LS14 6UQ.

Accepted for publication 22 September 1983. activities during the period of recording and noted these activities in a diary. An Oxford medical systems analyser was used for analysis. All abnormalities were recorded, and routine printouts were obtained for 10 seconds every four hours even when no irregularity was noted. Any unsatisfactory tapes were discarded and rerecorded.

For each boy the following observations were made: (a) maximum and minimum heart rates while awake and asleep and the rhythm present at these extremes; (b) variations in PP interval whether phasic or sudden (in 25 cases in which short sinus pauses occurred (PP interval exceeding previous PP interval by $50-90 \%$ ) each PP interval was plotted against the preceding PP interval according to the method of Schamroth and Dove ${ }^{9}$ to distinguish sinus arrhythmia from type I second degree sinoatrial block); (c) variations in $\mathrm{P}$ wave morphology and PR interval; (d) first degree atrioventricular block defined as a PR interval of $0.20 \mathrm{~s}$ or more; (e) second degree atrioventricular block, whether type I or type II; and (f) the presence and number of ventricular or supraventricular extrasystoles or episodes of paroxysmal tachycardia.

\section{Results}

Seventeen boys were in sinus rhythm, without change in $\mathrm{P}$ wave morphology or sinus pauses, throughout the period of the recording. 


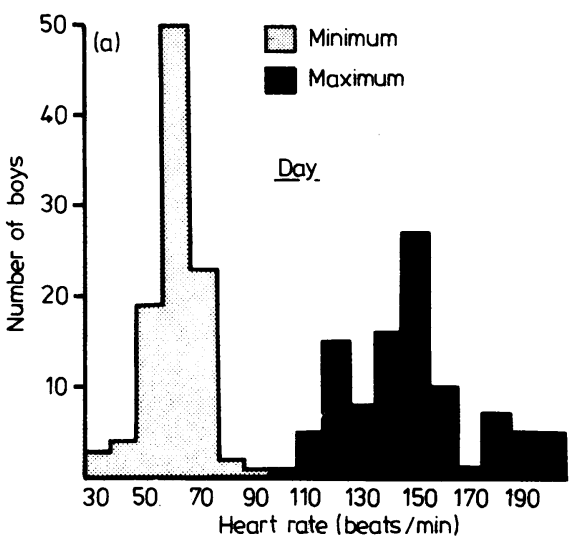

HEART RATES

The lowest and highest recorded heart rates during sleep and while awake are shown (Figure). Maximal heart rates of 180 to 200 beats $/ \mathrm{min}$ were recorded in 17 boys who undertook strenuous exercise. All were in sinus rhythm. The lowest sinus rate recorded during sleep was 35 beats/min.

\section{VARIATION IN PP INTERVAL}

A gradual increase and subsequent decrease in PP interval (sinus arrhythmia) were present at some time during all recordings and were particularly noticeable at night when sinus rates were slower. The presence of sinus arrhythmia made the diagnosis of type I (Wenckebach) sinoatrial block difficult. Although short sinus pauses occurred in 25 boys, using the method of Schamroth and Dove, 9 we were unable to identify any who had unequivocal evidence of type I second degree sinoatrial block.

Sudden prolongation of the PP interval occurred in 16 recordings. In 15 , the prolonged PP interval exceeded the preceding PP interval by more than $110 \%$, a finding that is compatible with either sinus arrest or temporary complete sinoatrial block. The maximum number of sinus pauses of this type in any boy was four in 24 hours, and the longest single pause was $1.88 \mathrm{~s}$. Sinus pauses were followed by single junctional escapes or periods of junctional rhythm in five boys and by a rhythm indistinguishable from the previous sinus rhythm in the remaining 10 .

In one boy the prolonged PP interval was exactly twice the preceding PP interval, which is compatible with either sinus arrest or type II second degree sinoatrial block.

\section{VARIATION IN P WAVE MORPHOLOGY AND PR INTERVAL}

Changes in $P$ wave morphology occurred in 46 boys; but in 20 the changes were minor, not associated with a change in PR interval, and probably resulted from changes in position. In 26 , however, a sudden major change in $\mathbf{P}$ wave axis always associated with a slower heart rate and shortening of the PR interval suggested a change in the site of the dominant pacemaker from the sinus node to the atrium or atrioventricular junction. In most cases these episodes of escape rhythm were short (range 5-60 s) and occurred intermittently over several hours during sleep. Four boys had longer continuous periods varying from seven minutes to six hours. In five boys the escape rhythm was preceded by a sinus pause, while in the remainder the precipitating event appeared to be a gradual slowing of the sinus rate.

\section{FIRST DEGREE ATRIOVENTRICULAR BLOCK}

Prolongation of the PR interval to $>0.2 \mathrm{~s}$ occurred in 12 boys, in nine during sleep only and in three both by day and by night. Six of the 12 boys developed Mobitz type I second degree atrioventricular block. In seven boys, the PR interval remained prolonged during sleep for periods of up to eight hours 47 minutes, whereas in four others the PR interval varied from cycle to cycle. One boy had prolongation of the PR interval to $0.2 \mathrm{~s}$ throughout the 24 hours.

\section{SECOND DEGREE ATRIOVENTRICULAR BLOCK}

Mobitz type I second degree block was found in 11 boys, in seven during sleep only and in four both while awake and during sleep. The number of episodes of type I block varied from 1 to 1045 in 24 hours, six boys each having more than 50 episodes within this period. Periods of $2: 1$ block for up to $15 \mathrm{~s}$ occurred in five boys after episodes of Mobitz type I block, and in one of these more advanced degrees of atrioventricular block occurred, including one episode of 5:1 block with an RR interval of $4.5 \mathrm{~s}$. The lowest heart rate in this boy was 23 beats/min during a period of 3:1 block. 
Mobitz type II atrioventricular block was seen on one occasion in one boy who also had first degree atrioventricular block intermittently throughout the 24 hours and episodes of Mobitz type I atrioventricular block on 92 separate occasions.

\section{VENTRICULAR EXTRASYSTOLES}

Ventricular extrasystoles were present in 41 boys. In 34 boys, the premature beats were isolated, but one had periods of bigeminy, and in three two consecutive extrasystoles occurred between one and four times. Ventricular tachycardia, defined as three or more consecutive depolarisations originating from the ventricle at a rate greater than 120 a minute, occurred in three boys. All had isolated ventricular extrasystoles throughout the 24 hours with two having, in addition, one episode of three consecutive extrasystoles and one having six consecutive extrasystoles.

Ventricular extrasystoles were of uniform morphology in 31 boys and multiform in the remaining 10. The total number of extrasystoles varied from one to 28000 in 24 hours; six had an average of more than one ventricular extrasystole an hour. In these six boys extrasystoles were absent at heart rates greater than 110 beats/min suggesting that they were suppressed by even mild exercise.

Supraventricular extrasystoles occurred in 44 boys and varied in number from one to 243 during the 24 hour period. Two consecutive supraventricular extrasystoles occurred at least once in two boys, but no episodes of supraventricular tachycardia were recorded.

\section{Discussion}

Previous reports of 24 hour electrocardiographic patterns in apparently normal neonates ${ }^{34}$ and children up to 13 years of age $^{56}$ have shown a variety of rhythm changes that might have been regarded as evidence of disease of the sinus node or atrioventricular conduction system had they been recorded on the standard resting electrocardiogram. ${ }^{1011}$ It is clear from these reports, however, that many of these rhythm changes are relatively common in an asymptomatic population, occurring in many individuals though not necessarily frequently. Such rhythm changes are, therefore, likely to be benign, although long term studies to verify this have yet to be performed.

The findings of our present study are similar to those of earlier reports in younger children, ${ }^{56}$ the main difference being in the greater frequency of ventricular extrasystoles in these older boys. In this respect, boys aged 14 to 16 years behave like the young adults reported by Brodsky $e t a^{7}$ and Clarke $e t$ $a l .8$ This tendency for the prevalence of ventricular extrasystoles to increase with age continues through the adult age groups so that by 65 years of age they occur in $80 \%$ of the population. ${ }^{12}$ The reasons for this remain uncertain. Neither coffee drinking nor cigarette smoking affected the prevalence of ventricular extrasystoles among the patients reported by Ekblom et al ${ }^{13}$ while studies of the influence of coronary artery disease have yielded conflicting results. ${ }^{13}{ }^{14}$ These reports are, however, largely concerned with extrasystoles induced by exercise, whereas in our patients the evidence from the observed heart rates and the boys' diaries suggested that they were suppressed by exercise. This finding agrees with those of previously reported studies in children. ${ }^{15}$

The benign nature of ventricular extrasystoles of uniform morphology in children with normal hearts has been shown by Jacobsen et al who followed 17 children with extrasystoles on a standard resting electrocardiogram for a mean period of $7 \cdot 2$ years. ${ }^{16}$ All remained asymptomatic, and in nine of the 17 extrasystoles were not present at the second examination, although the value of this latter observation on a standard resting electrocardiogram is limited. For multiform extrasystoles the position is less clear. As our present and previous studies 5 show, they are not uncommon in childhood. Nevertheless, one of the four children with multiform ventricular extrasystoles reported by Jacobsen et al ${ }^{16}$ died suddenly, and further information regarding the importance of this type of extrasystole in this age group is needed.

First degree atrioventricular block and Mobitz type I second degree block (Wenckebach) have been noted previously in both younger children ${ }^{56}$ and healthy young adults ${ }^{7}$ and are particularly common in trained athletes. ${ }^{1718}$ Schamroth has suggested ${ }^{19}$ that atrioventricular block in athletes is a manifestation of increased vagal activity, and vagal stimulation has been shown to induce Mobitz type I atrioventricular block in some individuals. ${ }^{20}$ All the boys in the present study took part in organised school games, and 17 undertook strenuous exercise out of school hours during the period of recording. Although we have no information about the degree of physical fitness of individual boys, the somewhat higher prevalence of first degree atrioventricular block and type I second degree block in these boys compared with that in 7 to 11 year old children ${ }^{6}$ might be accounted for by their participation in sports. However, in the absence of more detailed information on the degree of training of individuals such a conclusion remains speculative. The importance of these arrhythmias is difficult to assess. While it seems improbable that such common arrhythmias could be implicated in sudden unexpected death in athletic young men ${ }^{21}$ progression of type I second degree atrioventricular block to complete heart block requiring a pacemaker has been 
reported, ${ }^{22}$ and this arrhythmia should not, therefore, be regarded as entirely benign. However, it is common, and in the absence of symptoms we would not regard it as an indication for further investigation, although long term follow up would be both prudent and of considerable interest. The preliminary report of Mehta and colleagues suggests that for most individuals with a structurally normal heart and Mobitz type I second degree atrioventricular block on the surface electrocardiogram the long term prognosis is good. ${ }^{23}$

Studies that have shown that Mobitz type I second degree atrioventricular block can progress to complete atrioventricular block ${ }^{22} 23$ have been based on small numbers of patients whose arrhythmia was found on a standard resting electrocardiogram, and the long term implications of this finding in this selected group may be different from that in individuals having only occasional episodes during a 24 hour period. This progression may, however, account for those children who present with complete heart block well beyond the neonatal period. When complete heart block is first recognised at this age it is usually regarded as congenital, ${ }^{24}$ but it is certainly possible that such children had partial atrioventricular block earlier in infancy.

Mobitz type II second degree atrioventricular block was recorded on one occasion in one boy and has previously been recognised in other apparently healthy individuals. ${ }^{818}$ As in these previously reported cases this boy also had numerous episodes of atrioventricular block, both first degree and second degree type I. Type II second degree block is not necessarily an ominous sign, but it is sufficiently uncommon to make its presence worrying, and the preliminary findings of Mehta et al ${ }^{23}$ suggest that most individuals with this type of arrhythmia will progress to third degree atrioventricular block or bradycardia with symptoms.

This study clearly shows that short pauses in sinus rhythm are common in boys of this age. Southall and colleagues found similar pauses occurring with even greater frequency in both neonates ${ }^{4}$ and younger children. ${ }^{6}$ The longest pause recorded by us was $1.88 \mathrm{~s}$, a finding almost identical to that reported in younger children. ${ }^{46}$ The mechanism responsible for these pauses remains uncertain. Although the presence of sinoatrial block can be inferred by careful measurement of atrial potentials on the surface electrocardiogram, a confident diagnosis may be achieved only by direct intracardiac recording of sinus node potentials. The universal presence of phasic variations in PP interval (sinus arrhythmia) in children ${ }^{4-6}$ together with automatic influences or the possible presence of unrecognised escape rhythms with a rate only slightly slower than the sinus rate 25 may confuse the measurement of the resting sinus cycle length, making a precise diagnosis of this arrhythmia from the surface electrocardiogram impossible. The method of Schamroth and Dove ${ }^{9}$ may be helpful in distinguishing sinus arrhythmia from type I second degree sinoatrial block, but we were unable to find convincing evidence for this latter arrhythmia in the study group. This may have been because the number of sinus pauses seen in any individual in 24 hours was small. For these reasons some workers have preferred to describe PP interval changes rather than to attempt a precise diagnosis in each case. We have also adopted this method. The results show that, although short sinus pauses occur in a high proportion of individuals, they do not appear to occur as frequently as in neonates ${ }^{4}$ and younger children. ${ }^{6}$

\section{CONCLUSION}

Most boys aged 14 to 16 years show variations from normal sinus rhythm. During sleep the sinus rate may fall as low as 30 beats/min, and during exercise heart rates of 200 beats/min may be reached. Escape beats or short periods of an escape rhythm probably originating in the atrioventricular junction are common, as are short sinus pauses. The mechanism responsible for these pauses is not clear, but we agree with Southall and colleagues ${ }^{6}$ that, within the limits described by those workers, they are likely to be benign. Ventricular extrasystoles, which are uncommon in healthy 7 to 11 year olds, ${ }^{6}$ become more common in adolescent boys, and by the age of 14 to 16 years they occur with a frequency similar to that in a young adult population. The clinical importance of these findings is clear. Many children present with symptoms that might be explained by a transient cardiac arrhythmia. Twenty four hour ambulatory monitoring of the electrocardiogram is a convenient means of evaluating these patients, ${ }^{2}$ but the results obtained should be interpreted in the knowledge of the range of rhythm changes that are seen in a normal population of similar age if unnecessary treatment of benign arrhythmias is to be avoided. Ideally, a precise correlation between symptoms and arrhythmia should be established before treatment is started. As Southall and colleagues ${ }^{6}$ have commented, similar caution should be exercised in the assessment of arrhythmias following cardiac surgery in children.

This work was supported by a grant from the British Heart Foundation.

\section{References}

1 Lipski J, Cohen L, Espinoza J, Motro M, Dack S, Donoso E. Value of Holter monitoring in assessing cardiac arrhythmias in symptomatic patients. Am f Cardiol 1976; 37: 102-6. 
2 Porter CJ, Gillete PC, McNamara DG. 24 hour ambulatory ECGs in the detection and management of cardiac dysrhythmias in infants and children. Pediatr Cardiol 1980; 1: 203-8.

3 Välimäki I. Tape recordings of the electrocardiogram in newborn infants. Section II. Long term ECG tape recordings of newborn infants. Acta Paediatr Scand [suppl] 1969; 199: 31-75.

4 Southall DP, Richards J, Mitchell P, Brown DJ, Johnstone PGB, Shinebourne EA. Study of cardiac rhythm in healthy newborn infants. Br Heart $\mathcal{F} 1980$; 43: 14-20.

5 Scott O, Williams GJ, Fiddler GI. Results of 24 hour ambulatory monitoring of the electrocardiogram in 131 healthy boys aged 10 to 13 years. Br Heart $\mathcal{F} 1980 ; 44$ : 304-8.

6 Southall DP, Johnston F, Shinebourne EA, Johnston PGB. 24 hour electrocardiographic study of heart rate and rhythm patterns in population of healthy children. Br Heart F 1981; 45: 281-91.

7 Brodsky M, Wu D, Denes P, Kanakis C, Rosen KM. Arrhythmias documented by 24 hour continuous electrocardiographic monitoring in 50 male medical students without apparent heart disease. Am $\mathcal{F}$ Cardiol 1977; 39: 390-5.

8 Clarke JM, Hamer J, Shelton JR, Taylor S, Venning GR. The rhythm of the normal human heart. Lancet 1976; ii: 508-12.

9 Schamroth L, Dove E. The Wenckebach phenomenon in sino-atrial block. Br Heart f 1966; 28: 350-8.

10 Radford DJ. Izukawa T. Sick sinus syndrome. Symptomatic cases in children. Arch Dis Child 1975; 50: 879 85.

11 Scott O, Macartney FJ, Deverall PB. Sick sinus syndrome in children. Arch Dis Child 1976; 51: 100-5.

12 Hinkle LE Jr, Carver ST, Stevens M. The frequency of asymptomatic disturbance of cardiac rhythm and conduction in middle aged men. Am $\mathcal{F}$ Cardiol 1969; 24: 629-50.

13 Ekblom B, Hartley LH, Day WC. Occurrence and reproducibility of exercise induced ventricular ectopy in normal subjects. Am $\mathcal{f}$ Cardiol 1979; 43: 35-40.

14 McHenry P, Morris SN, Kavalier M, Jordan JW. Comparative study of exercise-induced ventricular arrhythmias in normal subjects and patients with documented coronary artery disease. Am $\mathcal{F}$ Cardiol 1976; 37: 609-16.

15 Rozanski JJ, Dimich I, Steinfeld L, Kupersmith J. Maximal exercise stress testing in evaluation of arrhythmias in children: results and reproducibility. $A m \mathcal{F}$ Cardiol 1979; 43: 951-6.

16 Jacobsen JR, Garson A Jr, Gillette PC, McNamara DG. Premature ventricular contractions in normal children. $\mathcal{F}$ Pediatr 1978; 92: 36-8.

17 Meytes I, Kaplinsky E, Yahini JH, Hanne-Paparo N, Neufeld HM. Wenckebach A-V block: a frequent feature following heavy physical training. Am Heart f 1975; 90: 426-30.

18 Viitasalo MT, Kala R, Eisalo A. Ambulatory electrocardiographic recording in endurance athletes. Br Heart $\mathcal{f}$ 1982; 47: 213-20.

19 Schamroth L. The disorders of cardiac rhythm. Oxford and Edinburgh: Blackwell Scientific Publications, 1971: 171 and 253.

20 Gambetta M, Denes P, Childers RW. Vagally induced second degree A-V block Mobitz type I, and the hyporeactive SA node. Chest 1972; 62: 152-5.

21 Roland JMA, Varghese PJ, Shematek J, Pieroni D. Sinus node dysfunction in young athletes: a possible cause of sudden death [Abstract]. Circulation 1975; 51 (suppl 2): 233

22 Young D, Eisenberg R, Fish B, Fisher JD. Wenckebach atrioventricular block (Mobitz type $\mathrm{I}$ ) in children and adolescents. Am $\mathcal{F}$ Cardiol 1977; 40: 393-9.

23 Mehta AV, Sanchez GR, Balian A, O'Riordan AC, Donner RM, Black IFS. Natural history of $2^{\circ}$ atrioventricular block in children: clinical and electrophysiological findings. Pediatr Cardiol 1982; 3: 351-2.

24 Olley PM, Cardiac arrhythmias. In: Keith JD, Rowe RD, Vlad P, eds. Heart disease in infancy and childhood. New York, Toronto, London: Macmillan, 1978: 290.

25 Ferrer MI. Significance of slow atrial rhythm. Am f Cardiol 1980; 46: 176-7. 